\title{
Melatonin and doxorubicin synergistically enhance apoptosis via autophagy-dependent reduction of AMPKa1 transcription in human breast cancer cells
}

\author{
Quynh Hoa Tran ${ }^{1,2,3}$, Dang Hieu Hoang ${ }^{1,3}$, Minhyeok Song ${ }^{1}$, Wonchae Choe ${ }^{1}$, Insug Kang ${ }^{1}$, Sung Soo Kim ${ }^{1}$ and Joohun Ha (D) ${ }^{1 凶}$ \\ (c) The Author(s) 2021
}

Doxorubicin is one of the most effective agents used to treat various cancers, including breast cancer, but its usage is limited by the risk of adverse effects, including cardiotoxicity. Melatonin, a natural hormone that functions as a major regulator of circadian rhythms, has been considered a supplemental component for doxorubicin due to its potential to improve its effectiveness. However, the mechanisms and biological targets of the combination of melatonin and doxorubicin with respect to cancer cell death are not well understood. In the present study, we found that melatonin synergized with doxorubicin to induce apoptosis of breast cancer cells by decreasing the expression of AMP-activated protein kinase a1 (AMPK a1), which acts as a critical survival factor for cancer cells. This cotreatment-induced reduction in AMPKa1 expression occurred at the transcriptional level via an autophagy-dependent mechanism. The synergistic effects of the combined treatment were evident in many other cancer cell lines, and melatonin was also highly effective in inducing cancer death when combined with other cancer drugs, including cisplatin, 5-fluorouracil, irinotecan, and sorafenib. AMPKa1 expression was decreased in all of these cases, suggesting that reducing AMPKa1 can be considered an effective method to increase the sensitivity of cancer cells to doxorubicin treatment.

Experimental \& Molecular Medicine (2021) 53:1413-1422; https://doi.org/10.1038/s12276-021-00675-y

\section{INTRODUCTION}

Breast cancer is one of the three most common cancers worldwide, along with lung and colon cancer $^{1,2}$. It is the most common malignancy and the leading cause of cancer-related death among females worldwide ${ }^{1}$. Breast cancer has become a global public health problem due to its complex etiology and poor response to treatment ${ }^{3}$. Doxorubicin, which is one of the most effective anthracycline agents used to treat breast cancer, can be used at all cancer stages as a curative agent for early and metastatic breast cancer ${ }^{2}$, even in patients with a high risk of relapse $^{4}$. For many years, doxorubicin has been used as a first-line treatment for not only most patients with breast cancer ${ }^{2}$ but also those with many types of soft tissue sarcoma ${ }^{5,6}$. Although doxorubicin is an effective chemotherapeutic agent against multiple types of malignancies, its application has been limited by the risk for cardiotoxicity ${ }^{7}$.

Melatonin ( $\mathrm{N}$-acetyl-5-methoxytryptamine) is an indolic compound that is synthesized and secreted mainly by the pineal gland and plays a central role in regulating circadian and seasonal biorhythms in humans ${ }^{8}$. Melatonin has shown chemotherapeutic potential in many forms of human cancer ${ }^{9-15}$ and can increase the efficacy of anticancer drugs (e.g., doxorubicin, cisplatin, epirubicin, and bleomycin) by regulating many different signaling pathways $^{16-22}$. Therefore, melatonin has been considered a potential supplementary component in chemotherapy to reduce the therapeutic doses and, thus, the adverse effects of anticancer drugs. However, the mechanisms by which melatonin synergizes with anticancer drugs are still unclear.

AMP-activated protein kinase (AMPK) is a heterotrimeric protein kinase composed of a catalytic subunit (a) and two regulatory subunits $(\beta$ and $\gamma)$ and plays a central role in the regulation of energy homeostasis ${ }^{23}$. The activity of AMPK is highly regulated at the protein level in response to changes in cellular energy status via allosteric regulation of adenine nucleotides (e.g., AMP, ADP, and ATP) and/or phosphorylation by upstream kinases, including LKB1 and calcium/calmodulin-dependent protein kinase kinase $\beta^{24}$. There are multiple isoforms of each subunit: the a1 catalytic subunit is ubiquitously expressed, whereas a2 shows a highly tissue-specific expression pattern that includes the heart, skeletal muscle, and liver ${ }^{25}$. The role of AMPK has been intensively investigated under various metabolic conditions, but its function and activity in the presence of anticancer drugs or melatonin remain largely unresolved. There is no consensus on the role of AMPK in regulating cell viability under doxorubicin treatment ${ }^{26-28}$, and our previous study suggested that the isoforms of AMPKa play opposing roles in determining cell fate under doxorubicin treatment ${ }^{29}$.

In the present study, we examined the potential of melatonin to enhance the efficacy of doxorubicin in breast cancer cell lines. We demonstrate that the combination of melatonin and doxorubicin

\footnotetext{
${ }^{1}$ Department of Biochemistry and Molecular Biology, Graduate School, College of Medicine, Kyung Hee University, Seoul 130-701, Republic of Korea. ${ }^{2}$ Department of Biotechnology, Ho Chi Minh city University of Food Industry, Ho Chi Minh, Vietnam. ${ }^{3}$ These authors contributed equally: Quynh Hoa Tran, Dang Hieu Hoang. ${ }^{\mathrm{m}}$ email: hajh@khu.ac.kr
}

Received: 23 December 2020 Revised: 29 June 2021 Accepted: 30 June 2021

Published online: 28 September 2021 
markedly induces apoptosis of breast cancer cells by reducing AMPKa1 messenger RNA (mRNA) at the transcriptional level in an autophagy-dependent manner. We also examined the effects of this combined treatment in various cancer cell lines and the effects of melatonin and several other anticancer drugs.

\section{MATERIALS AND METHODS Reagents and antibodies}

Dulbecco's modified Eagle's medium (DMEM) and RPMI-1640 were purchased from Gibco (Grand Island, NY, USA). The breast cancer (MCF7, MDA-MB157, MDA-MB231), colorectal carcinoma (HTC116), lung adenocarcinoma (A549), gastric adenocarcinoma (AGS), hepatocellular carcinoma (HepG2, Huh7), and mouse embryonic fibroblast (MEF) cell lines used in this study were purchased from American Tissue Culture Collection (ATCC, Manassas, VA, USA). Ampka1 $1^{-1-}$ knockout MEFs were a generous gift from Dr. B. Viollet (INSERM, Paris, France). Fetal bovine serum (FBS) was purchased from Corning (Corning, NY, USA). Antibodies against phosphoacetyl-CoA carboxylase $a-S_{-} r^{79}$, cleaved caspase-3, caspase-7, cleaved caspase-7, poly (ADP-ribose) polymerase (PARP), p62, and microtubuleassociated protein $1 \mathrm{~A} / 1 \mathrm{~B}$-light chain $3 \mathrm{~B}(\mathrm{LC}-3 \mathrm{~B})$ were purchased from Cell Signaling Technology (Danvers, MA, USA). Antibodies against AMPKa1 and AMPKa2 were obtained from R\&D Systems (Minneapolis, MN, USA). Antibodies against human influenza hemagglutinin and PARP were obtained from Santa Cruz Biotechnology (Santa Cruz, CA, USA). Anti$\beta$-actin was purchased from Proteintech (Rosemont, IL, USA). Horseradish peroxidase (HRP)-conjugated rabbit anti-mouse and goat anti-rabbit secondary antibodies were purchased from Thermo Fisher (Waltham, MA, USA). G-418 (Geneticin) was purchased from Duchefa Biochemie (Haarlem, Netherlands). Melatonin (5-methoxy- $N$-acetyltryptamine), doxorubicin, irinotecan, cisplatin, and 5-fluorouracil (5-FU) were purchased from Sigma-Aldrich (St. Louis, MO, USA). Sorafenib and 3-methyladenine (3-MA) were purchased from Santa Cruz Biotechnology (Dallas, TX, USA). Small interfering RNAs (siRNAs) targeting AMPKa1 and ATG5 and nontargeting siRNAs (si-ctl) were purchased from GenePharma (Zhangjiang Hi-Tech Park, Shanghai, China). The siRNA transfection reagent INTERFERin ${ }^{\circ}$ was purchased from Polyplus-transfection (New York, NY, USA). The DNA transfection reagent TransIT-X2 ${ }^{\circledR}$ was obtained from Mirus Bio LLC (Madison, WI, USA).

\section{Cell cultures}

MDA-MB-157, MDA-MB-231, HepG2, and MEFs were maintained in DMEM. Huh7, MCF-7, HCT116, A549, and AGS were cultured in RPMI-1640. All media were supplemented with $10 \%$ FBS and antibiotics, and cultures were incubated at $37{ }^{\circ} \mathrm{C}$ in humidified air containing $5 \% \mathrm{CO}_{2}$.

\section{Plasmid construction and transfection}

The promoter region of the human AMPKa1 gene $(\sim 1.7 \mathrm{~kb})$ was amplified by PCR and inserted into the PGL3-basic reporter vector. MDA-MB-157 and MCF-7 cells were cultured in a growth medium and transfected with plasmids using TransIT- $\mathrm{X} 2^{\circledR}$ transfection reagent according to the manufacturer's instructions (Mirus, Madison, WI, USA). All plasmid constructs were confirmed by sequencing.

\section{Establishment of stable overexpression cell lines}

MDA-MB-157 and MCF-7 cells were transfected with pcDNA3-Ha-AMPKa1 or pCDNA3 vector (negative control). After $24 \mathrm{~h}$, successfully transfected clones were selected with $\mathrm{G}-418(200 \mu \mathrm{g} / \mathrm{ml})$ for 2 weeks.

\section{RNA isolation and RT-PCR}

RNA samples were extracted using TRIzol (Life Technologies, Grand Island, NY, USA). Reverse transcription-polymerase chain reaction (RT-PCR) was performed with $1 \mu \mathrm{g}$ of extracted RNA by using a complementary DNA (cDNA) Synthesis Kit (Doctor Protein, Seoul, Korea). PCR was performed using Taq polymerase, primers against AMPKa1 or glyceraldehyde 3-phosphate dehydrogenase (GAPDH), and $1 \mu \mathrm{g}$ of CDNA. The human AMPKa1 and GAPDH primer sequences were as follows: AMPKa1, forward (5'-CATGAAG AGGGCCACAATCA-3') and reverse (5'-GGGCTTGTCGCCAAATAGAA-3'); and GAPDH, forward (5'-TGGGCTACACTGAGCACCAG-3') and reverse (5'-AC CACCCTGTTGCTGTAGCC- $3^{\prime}$ ). The primers for the PCR analysis of mouse AMPKa1 and GAPDH were as follows: AMPKa1, forward (5'-GATCGGCCACTAC ATCCTGG-3') and reverse (5'-GATGTGAGGGTGCCTGAACA-3'); and GAPDH, forward (5'-GGAAGGGCTCATGACCACAGTCC-3') and reverse (5'-CGACGGACACATTGGGGGTAGGA-3'). The level of each mRNA was normalized to that of GAPDH.

\section{Reporter gene assay}

Cells were seeded in 12 -well culture plates at $5 \times 10^{4}$ cells/well and incubated for $24 \mathrm{~h}$. Plasmids were transfected into cells with TransIT-X2 ${ }^{\circledR}$ according to the manufacturer's instructions. After $24 \mathrm{~h}$ of transfection, cells were treated with the indicated drugs for $24 \mathrm{~h}$. Then, luciferase activity was determined by mixing $20 \mu \mathrm{l}$ of cell extract with $80 \mu \mathrm{l}$ of luciferase assay reagent (Promega, Madison, WI, USA), and relative light units were measured using a Synergy HTX luminescence plate reader (BioTek, Winooski, VT, USA).

\section{Cell death assay}

Apoptotic cells were detected with a MUSE Annexin V \& Dead Cell Kit and a Muse cell analyzer (both from Merck Millipore, Danvers, MA, USA) according to the manufacturer's instructions. The apoptotic ratio was determined by identifying four populations: (i) cells not undergoing detectable apoptosis, which were Annexin $\mathrm{V}(-)$ and 7-amino actinomycin $\mathrm{D}(\mathrm{AAD})(-)$; (ii) early apoptotic cells, which were Annexin V (+) and 7-AAD (-); (iii) late apoptotic cells, which were Annexin V (+) and 7-AAD (+); and (iv) cells that had died through a nonapoptotic pathway, which were Annexin $V(-)$ and 7-AAD (+).

\section{Western blot analysis}

Cells were washed with phosphate-buffered saline and lysed in lysis buffer $(150 \mathrm{mM} \mathrm{NaCl}$, Tris- $\mathrm{HCl}, 50 \mathrm{mM}, \mathrm{pH} 7.4,1.0 \%$ Triton X-100, 0.1\% sodium dodecyl sulfate (SDS), $5 \mathrm{mM}$ EDTA, $10 \mathrm{mM} \mathrm{NaF}$, and $2.0 \mathrm{mM} \mathrm{Na} \mathrm{VO}_{4}$ ) containing a protease inhibitor cocktail tablet (sc-29130; Santa Cruz Biotechnology). The lysates were cleared via centrifugation $(21,000 \times g$ at $4{ }^{\circ} \mathrm{C}$ for $15 \mathrm{~min}$ ), and the supernatant from each sample was collected. The protein concentration was determined using Bradford's solution at a ratio of 1:100 (cell lysate:Bradford solution). Equal amounts of protein $(10 \mu \mathrm{g})$ were subjected to SDS-polyacrylamide gel electrophoresis through $8-13 \%$ gels. Proteins were transferred to a polyvinylidene fluoride membrane, and the membrane was washed with TBST solution $(150 \mathrm{mM} \mathrm{NaCl} ; 10 \mathrm{mM}$ Tris$\mathrm{HCl}, \mathrm{pH} 7.6$; and $0.1 \%$ Tween-20). The membrane was blocked with $5 \%$ bovine serum albumin in TBST solution for $1 \mathrm{~h}$, washed with TBST, and incubated with the appropriate primary antibody $(1: 1000)$ overnight at $4{ }^{\circ} \mathrm{C}$. The membrane was then washed and incubated with HRP-conjugated secondary antibody $(1: 10,000)$ for $1 \mathrm{~h}$ at room temperature. The Western blot bands were visualized using an enhanced chemiluminescence solution (Bio-Rad, Hercules, CA, USA). The signal intensity of primary antibody binding was quantified and normalized to that of the loading control ( $\beta$-actin).

\section{Statistical analysis}

The results are expressed as the means \pm SEM. Each experiment was repeated at least three times with three samples, and the data were analyzed by two-tailed Student's $t$ test. $P<0.05$ was considered significant. $P$ values are indicated in the figures as follows: ${ }^{* * *} p<0.005$, ${ }^{* *} p<0.01$, and ${ }^{*} p<0.05 ;$ n.s. indicates not significant.

\section{RESULTS}

Melatonin synergizes with doxorubicin to promote apoptosis and decrease AMPKa1 expression in breast cancer cells Initially, we examined the effects of doxorubicin and melatonin either alone or in combination on the protein levels of AMPKa1 and apoptotic markers (cleaved caspase-7 and cleaved PARP) in breast cancer cells. Doxorubicin treatment of MDA-MB-157 breast cancer cells increased the cleavage of caspase-7 and PARP and decreased the level of AMPKa1 in a dose-dependent manner (Fig. 1a). By contrast, melatonin showed no significant effect on these parameters at doses up to $3 \mathrm{mM}$ (Fig. 1b). We then tested the combination of $1 \mu \mathrm{M}$ doxorubicin and $3 \mathrm{mM}$ melatonin, which were dosages that had no significant effect when applied alone. This combined treatment resulted in a significant induction of apoptosis and reduction in AMPKa1 expression in several different breast cancer cell lines, including MDA-MB-157, MDA-MB-231, and 
a

MDA-MB157
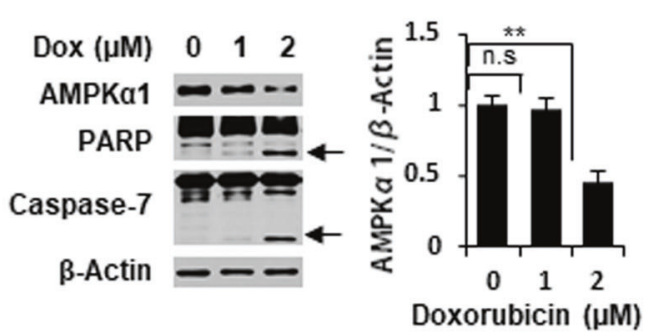

b

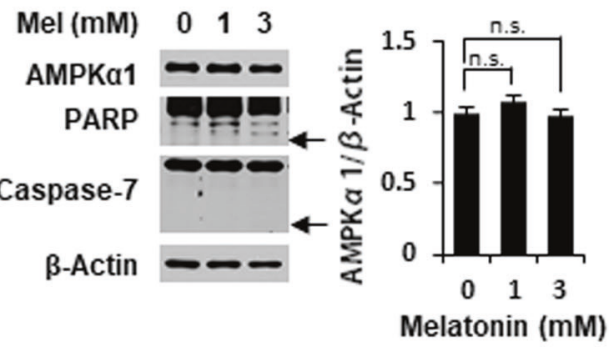

C

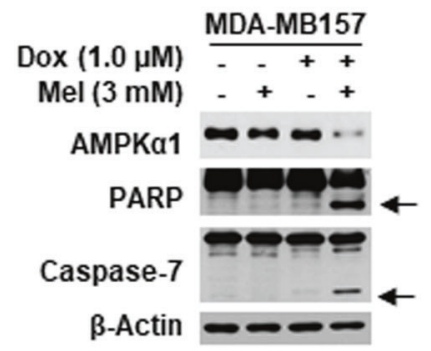

d
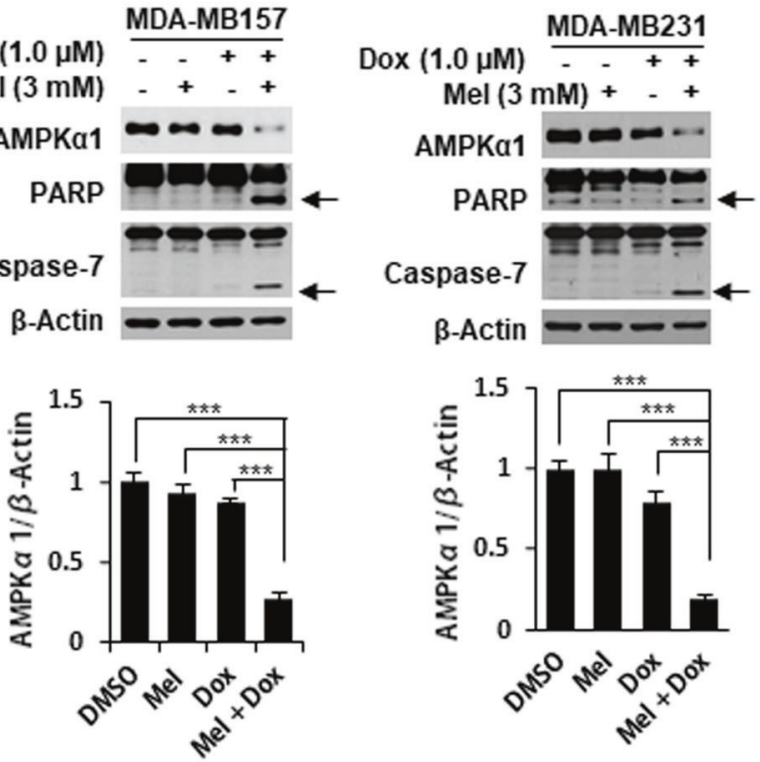

e
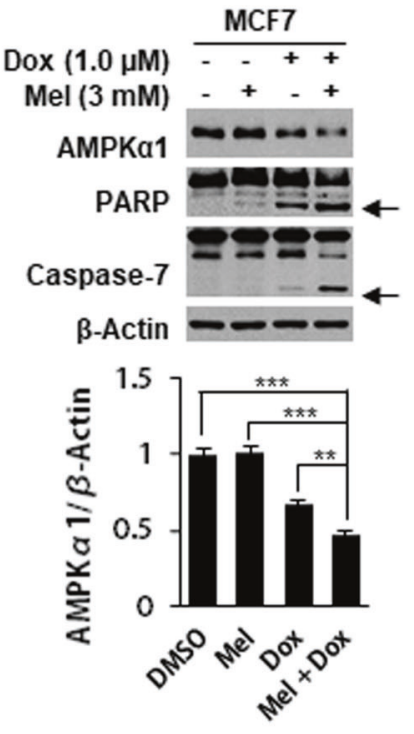

MDA-MB157

f
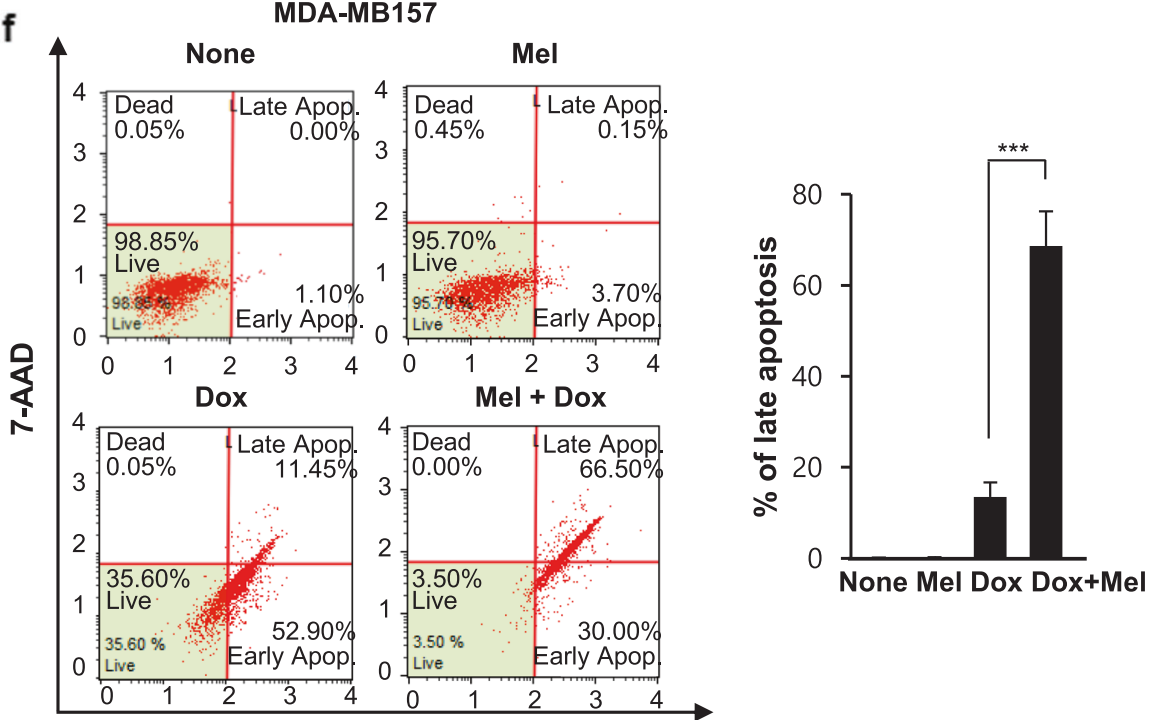

Annexin V

Fig. 1 The effects of melatonin and doxorubicin on apoptosis and the level of AMPKa1 in breast cancer cells. MDA-MB-157 breast cancer cells were treated with the indicated concentrations of doxorubicin (a) or melatonin (b) for $24 \mathrm{~h}$. MDA-MB-157 (c), MDA-MB-231 (d) and MCF-7 (e) cells were pretreated with $3 \mathrm{mM}$ melatonin for $2 \mathrm{~h}$, and then $1 \mu \mathrm{M}$ doxorubicin was added for $24 \mathrm{~h}$. The expression levels of AMPK 1 , caspase-7, and PARP were examined via Western blot analyses, and the band intensity of AMPK 1 was quantified. The arrow indicates the cleaved form (a-e). The populations of 7-ADD and Annexin V double-positive MDA-MB-157 cells were measured by Muse cell analyzer (f). Dox doxorubicin, Mel melatonin. ${ }^{* *} p<0.005 ;{ }^{* *} p<0.01 ;{ }^{*} p<0.05 ;$ n.s. not significant. 
MCF-7 cells (Fig. 1c-e), whereas the same dose of doxorubicin with $1 \mathrm{mM}$ melatonin did not show a synergistic effect. The synergistic effect of this combined treatment on apoptosis was further demonstrated via fluorescence-based analysis of 7-ADD and Annexin V double-positive cells (Fig. 1f). These results suggest that the combined treatment with doxorubicin and melatonin is highly effective for inducing apoptosis in breast cancer cells. Our data also indicate that the level of AMPKa1 is inversely correlated with the degree of apoptosis in response to this treatment.

\section{Knockdown of AMPKa1 promotes doxorubicin-induced apoptosis of breast cancer cells}

We next examined the role of AMPKa1 in regulating the fate of breast cancer cells under doxorubicin treatment, as this is poorly understood. Knockdown of AMPKa1 via siRNA in MDA-MB-157 and MCF-7 cells resulted in a marked augmentation of doxorubicin-induced apoptosis (Fig. 2a), whereas overexpression of AMPKa1 rendered these breast cancer cells highly resistant to doxorubicin-induced apoptosis (Fig. 2b, c). In accordance with the results of AMPKa1 knockdown, the combined treatment with doxorubicin and the AMPK inhibitor compound $C$ synergistically induced the apoptosis of MDA-MB-157 cells (Fig. 2d). Moreover, AMPKa1-knockout MEFs (Ampka1 ${ }^{-l-}$ ) were more sensitive to doxorubicin than were wild-type MEFs (Fig. 2e, f). These results suggest that AMPKa1 plays a critical role in protecting breast cancer cells against doxorubicin-induced cytotoxicity, further indicating that AMPKa1 is a cellular factor that contributes to determining cellular susceptibility to doxorubicin.

\section{Melatonin synergistically enhances the chemotherapeutic effect of doxorubicin via induction of autophagy}

The role of autophagy in the regulation of doxorubicin sensitivity among cancer cells, along with the relevant underlying mechanisms, has become the subject of substantial interest ${ }^{30,31}$. Here, we observed that treatment of MDA-MB-157 cells with $2 \mu \mathrm{M}$ doxorubicin, which induced apoptosis and reduced the level of AMPKa1, also induced autophagy, as indicated by an increase in the level of LC-3B and a decrease in the level of p62/SQSTM1 (p62) (Fig. 3a). Furthermore, the AMPKa1 level was recovered in these cells by adding the autophagy inhibitor 3-MA (Fig. 3a), suggesting that the downregulation of AMPKa1 is tightly associated with autophagy induced by doxorubicin treatment. To further study this apparent association, we blocked doxorubicin-induced autophagy via siRNA-mediated knockdown of autophagy-related gene 5 (Atg5) in MDA-MB-157 and MCF-7 cells and examined the level of AMPKa1 and the degree of apoptosis (Fig. 3b). Atg5 is well known to play a critical role in inducing autophagy. Our present results showed that doxorubicin-induced apoptosis was significantly blocked and that the level of AMPKa1 was not reduced when autophagy was blocked by Atg5 knockdown (Fig. 3b). Similar phenomena were observed in Atg5-knockout MEFs $\left(\right.$ Atg $^{-1-}$ ) (Fig. 3c). Moreover, the combination of $1 \mu \mathrm{M}$ doxorubicin and $3 \mathrm{mM}$ melatonin robustly induced autophagy in MDAMD-157 breast cancer cells (Fig. 3d). Taken together, these results suggest that doxorubicin treatment induces autophagy to contribute to breast cancer cell apoptosis and that the expression of AMPKa1, which protects against doxorubicin-induced cytotoxicity, is reduced in an autophagy-dependent manner.

\section{Melatonin and doxorubicin synergistically inhibit AMPKa1 transcription}

In response to various nutritional and stressful stimuli, AMPK enzyme activity is rapidly regulated via allosteric regulation by AMP, ADP, and ATP, as well as by phosphorylation by upstream kinases; meanwhile, the protein level of AMPKa1 remains relatively constant $^{23}$. Given our finding that the level of AMPKa1 was dramatically reduced by doxorubicin, we next examined whether AMPKa1 protein stability was affected by doxorubicin treatment.
MDA-MD-157 breast cancer cells were incubated with the protein translation inhibitor cycloheximide in the presence or absence of doxorubicin, and we monitored the AMPKa1 protein level for $24 \mathrm{~h}$ (Fig. 4a). We did not observe a significant difference in the half-life of AMPKa1 protein under these conditions (Fig. 4a). Notably, however, our subsequent RT-PCR analysis revealed that the AMPKa1 mRNA level was significantly reduced in response to treatment with $2 \mu \mathrm{M}$ doxorubicin (Fig. $4 \mathrm{~b}$ ), but not with melatonin (Fig. 4c). Melatonin treatment also potentiated the effect of $1 \mu \mathrm{M}$ doxorubicin (which had no significant effect when applied alone) on the AMPKa1 mRNA level (Fig. 4d). We further examined the half-life of AMPKa1 mRNA under doxorubicin treatment by incubating cells with the transcription inhibitor actinomycin $D$ in the presence or absence of $2 \mu \mathrm{M}$ doxorubicin. Our results showed that doxorubicin did not affect the half-life of AMPKa1 mRNA (Fig. 4e). To examine whether doxorubicin regulates the transcriptional activity of AMPKa1, we cloned a human AMPKa1 promoter fragment $(\sim 1.7 \mathrm{~kb})$ into the pGL3 luciferase reporter vector and transfected this construct into MDA-MD-157 breast cancer cells. In accordance with our findings with regard to the mRNA levels of AMPKa1 (Fig. 4b-d), doxorubicin treatment inhibited AMPKa1 promoter activity in a dose-dependent manner (Fig. 4f). Although treatment with either $1.0 \mu \mathrm{M}$ doxorubicin or $3.0 \mathrm{mM}$ melatonin alone had relatively little effect, the combination treatment markedly inhibited AMPKa1 promoter activity (Fig. 4f). The addition of 3-MA (an autophagy inhibitor) effectively blocked the effect of either $2 \mu \mathrm{M}$ doxorubicin or the combination treatment comprising $1.0 \mu \mathrm{M}$ doxorubicin and $3.0 \mathrm{mM}$ melatonin on the promoter activity (Fig. 4f) and mRNA level (Fig. 4g) of AMPKa1. Moreover, the inhibitory effect of doxorubicin on AMPKa1 mRNA levels was not observed in Atg $^{-1-}$ MEFs (Fig. 4h). Collectively, these results suggest that doxorubicin decreases AMPKa1 mRNA at the transcriptional level in an autophagy-dependent manner and that melatonin potentiates this effect.

\section{Melatonin and doxorubicin cotreatment synergistically reduces AMPKa1 in various cancer cells}

Doxorubicin chemotherapy is prominently used for not only breast cancer ${ }^{2}$ but also many other forms of cancer ${ }^{5,6}$. Accordingly, we next examined the effects of doxorubicin and melatonin on AMPKa1 levels in other cancer cell lines, including hepatocellular carcinoma (HepG2, Huh7), colorectal carcinoma (HCT116), lung adenocarcinoma (A549), and gastric adenocarcinoma (AGS) cell lines. Similar to our findings in breast cancer cells, doxorubicin treatment reduced AMPKa1 expression and induced autophagy and apoptosis in a dose-dependent manner (Fig. 5a), and combined treatment with $3.0 \mathrm{mM}$ melatonin and $1.0 \mu \mathrm{M}$ doxorubicin markedly reduced AMPKa1 mRNA levels and induced autophagy and apoptosis in all of the tested cancer cell lines (Fig. 5b).

\section{The combination of melatonin with several different} anticancer drugs synergistically reduce AMPKa1

Finally, we examined the effects of combination treatment of melatonin with several other anticancer drugs, including cisplatin (Figs. 6a), 5-FU (Fig. 6b), irinotecan (Fig. 6c), and sorafenib (Fig. 6d), on AMPKa1 levels. Our results showed that melatonin potentiated the apoptosis-inducing effects of these anticancer drugs and that this effect was associated with significant reductions in the mRNA level of AMPKa1 (Fig. 6e-h).

\section{DISCUSSION}

The goal of chemotherapy is to promote cancer cell death without damaging noncancerous cells. Doxorubicin is an essential chemotherapeutic reagent for the treatment of breast cancer ${ }^{2,4}$ as well as of many other forms of cancer ${ }^{5,6}$, but its clinical 
a

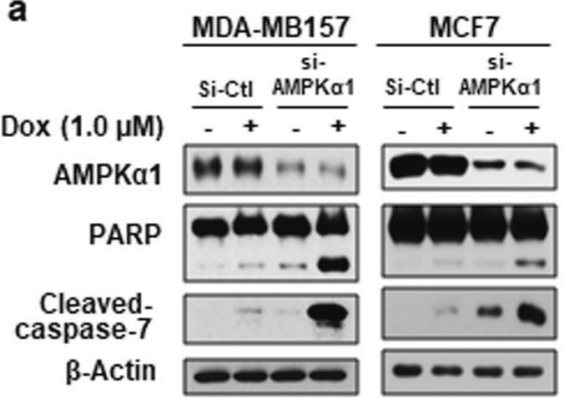

b

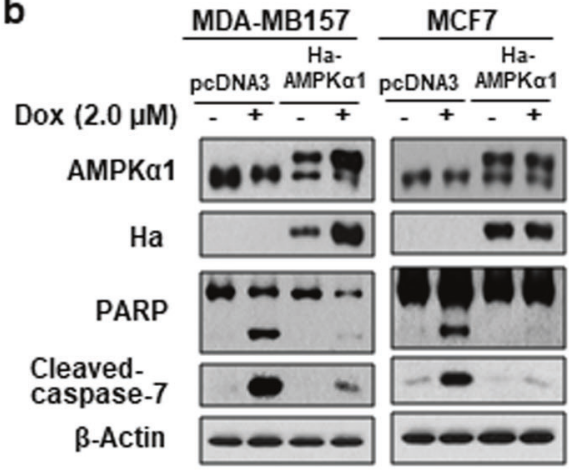

MDA-MB157 MCF7
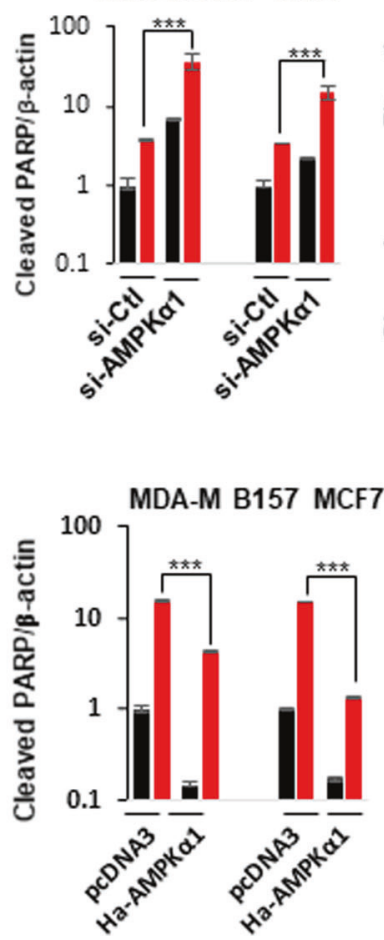

anone Dox

MDA-MB157 MCF7

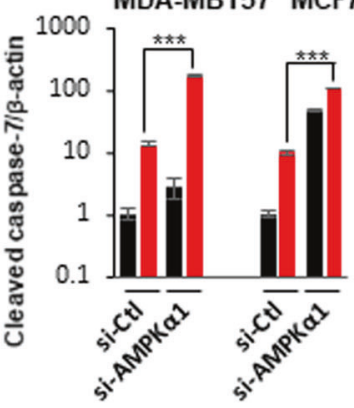

None Dox

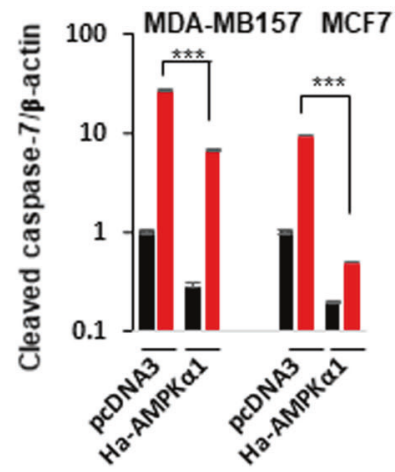

c MDA-MB157

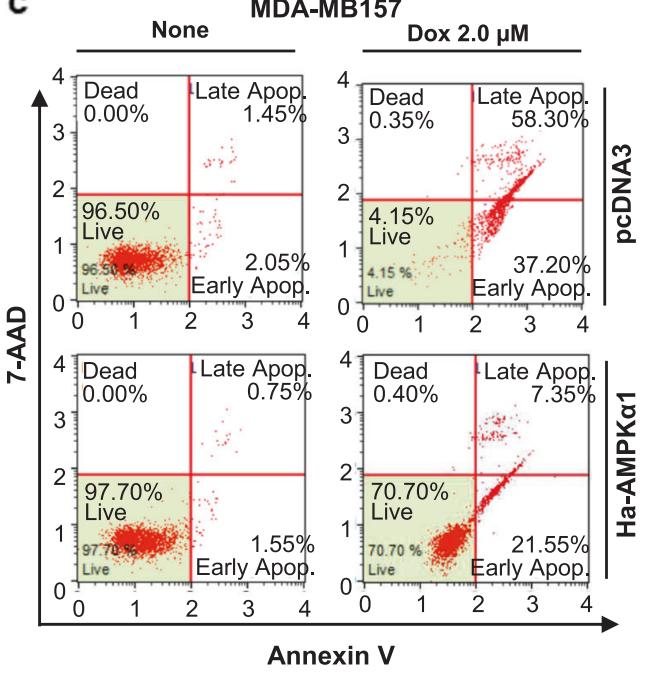

e

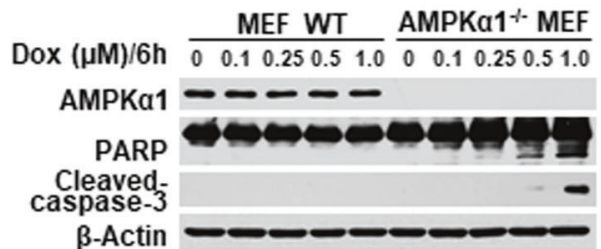

d None Dox

MDA-MB157

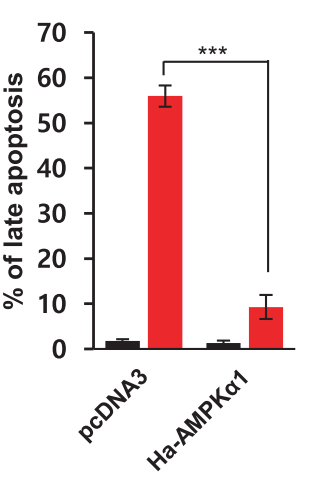

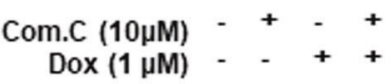

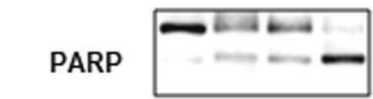

Cleaved
aspase-3

Cleaved

Caspase-7

$\beta$-actin $\ldots \ldots-\infty$

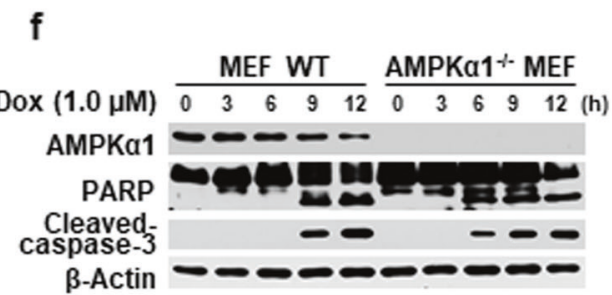

Fig. 2 Knockdown of AMPKa1 promotes doxorubicin-induced apoptosis of breast cancer cells. MDA-MB-157 and/or MCF-7 breast cancer cells were transfected with siRNA targeting AMPK $\alpha 1$ (a) or an expression vector overexpressing HA-tagged AMPK 1 (b, c). The cells were then incubated with the indicated concentrations of doxorubicin for $24 \mathrm{~h}$. Western blot analyses $(\mathbf{a}, \mathbf{b})$ or analyses of 7-ADD and Annexin $\mathrm{V}$ doublepositive MDA-MB-157 cells (c) were performed. MDA-MB-157 cells were treated with doxorubicin and the AMPK inhibitor compound C for $24 \mathrm{~h}$ (d). Wild-type and $A M P K a 1^{-I-}$ MEFs were treated with the indicated concentrations of doxorubicin for $6 \mathrm{~h}(\mathbf{e})$ or with $1 \mu \mathrm{M}$ doxorubicin for the indicated time periods (f). The expression levels of AMPK $\alpha 1$, PARP, and cleaved caspase- 3 were then examined via Western blot analyses. si-CtI nonspecific siRNA, si-AMPK $\alpha 1$ siRNA targeting AMPK $\alpha 1$, Com. C compound C. ${ }^{* * *} p<0.005 ;{ }^{* *} p<0.01 ;{ }^{*} p<0.05 ;$ n.s. not significant. 
a

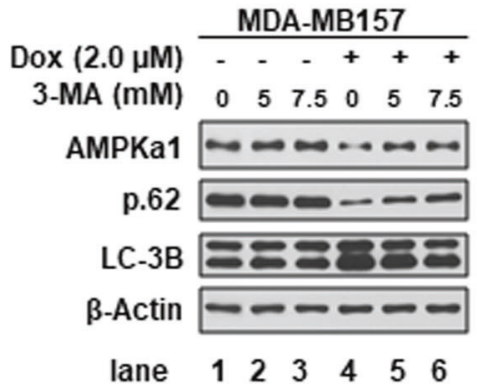

b

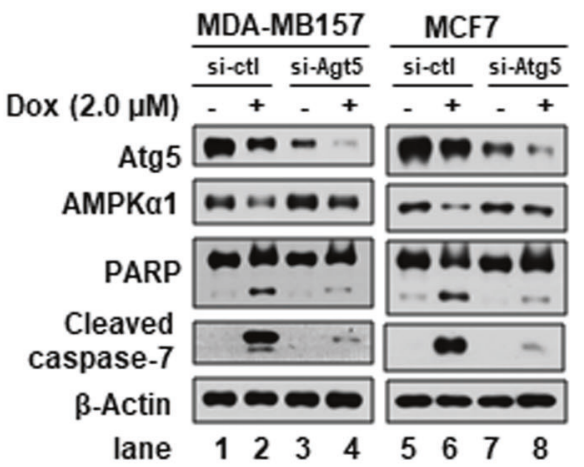

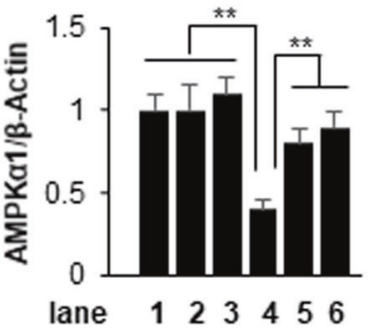

C
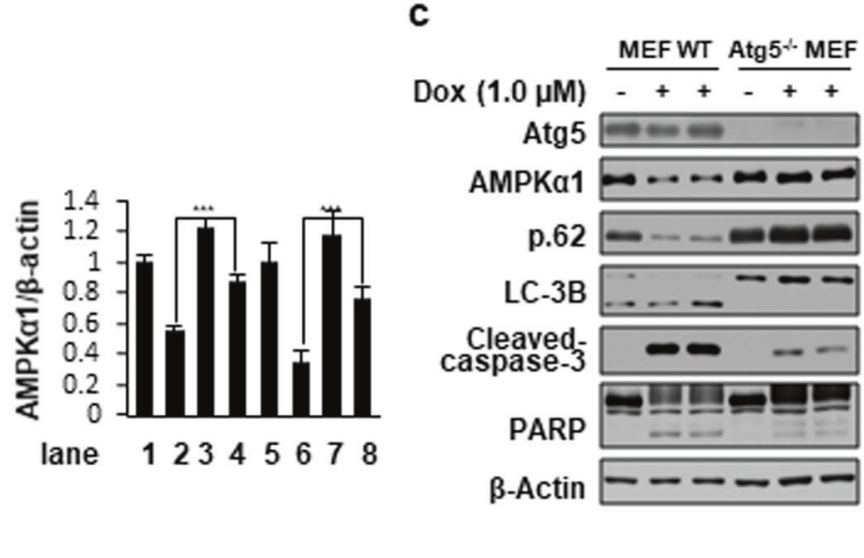

d

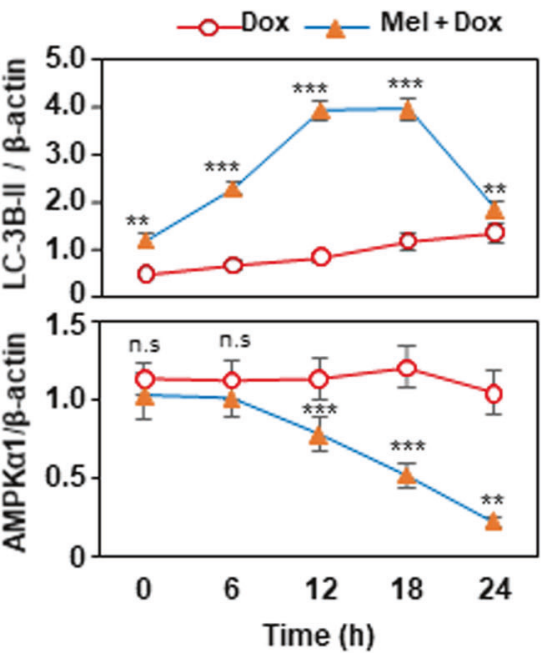

Fig. 3 Melatonin and doxorubicin synergistically enhance autophagy in breast cancer cells. a MDA-MB-157 cells were pretreated with the indicated concentrations of 3-MA for $30 \mathrm{~min}$ and then cultured in the presence or absence of doxorubicin for $24 \mathrm{~h}$. b MDA-MB-157 and MCF-7 cells were transfected with siRNA targeting Atg5 (si-Atg5) and then treated with doxorubicin for $24 \mathrm{~h}$. c Wild-type and Atg5 ${ }^{-1-}$ MEFs were treated with doxorubicin for $12 \mathrm{~h}$. d MDA-MB-157 cells were treated with doxorubicin for the indicated durations in the presence or absence of melatonin $(3 \mathrm{mM})$. Under these conditions, the expression levels of target proteins were examined via Western blot analyses. ${ }^{* * *} p<0.005$; ${ }^{* *} p<0.01 ;{ }^{*} p<0.05$; n.s. not significant.

application has been impeded by its risks for adverse effects, including cardiotoxicity ${ }^{7}$. Therefore, researchers are urgently seeking to improve the therapeutic efficacy of doxorubicin therapy while minimizing the adverse effects. Melatonin appears to be one of the most effective supplementary components that can satisfy this requirement, as it can reestablish the sensitivity of breast tumors to doxorubicin while protecting against doxorubicin-induced cardiotoxicity ${ }^{32}$. Combined treatment comprising doxorubicin and melatonin was previously demonstrated to have synergistic effects in inducing apoptosis in hepatoma ${ }^{17}$ and breast cancer $^{33}$ cells. Melatonin also reportedly showed cardioprotective effects against several anticancer drugs ${ }^{34}$.
Although multiple signaling pathways have been studied with the aim of understanding how melatonin synergizes with anticancer drugs, including doxorubicin ${ }^{17-22}$, the precise mechanisms remain highly unclear. In the present study, we observed that the combination of melatonin and doxorubicin reduced the level of AMPKa1, which acts as a critical survival factor for cancer cells; this phenomenon was seen in many different cancer cells, including breast cancer cells, and was also evident in cells subjected to combined treatment with melatonin and other cancer drugs. We, therefore, suggest that reducing AMPKa1 levels can be further developed as an adjuvant therapy to increase the sensitivity of cancer cells toward doxorubicin and thereby reduce its adverse 
a

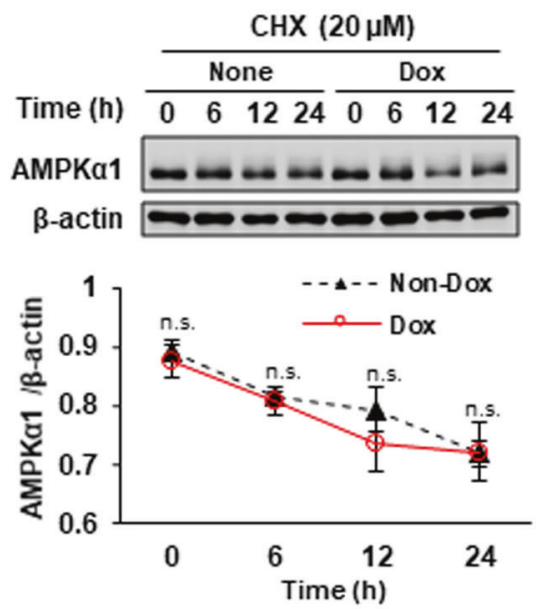

d
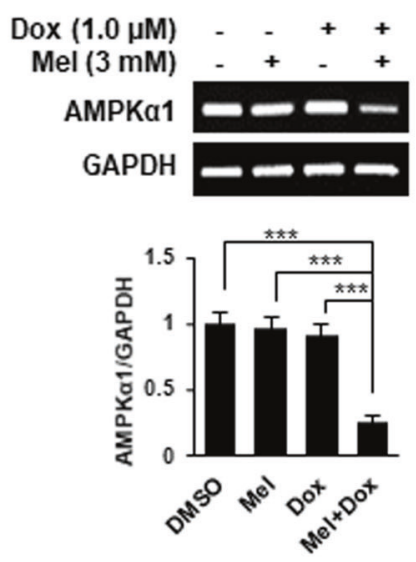

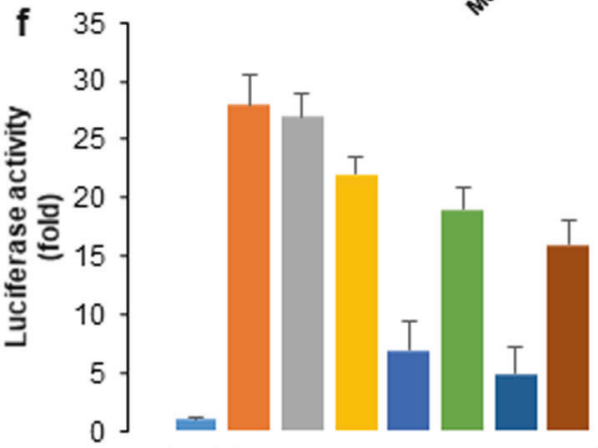

pGL3 vector AMPK $\alpha 1$ promoter

- None

Mel $3.0 \mathrm{mM}$

$=\mathrm{Dox} 1.0 \mu \mathrm{M}$

- Mel $3.0 \mathrm{mM}$ + Dox $1 \mu \mathrm{M}$

= Mel $3.0 \mathrm{mM}$ + Dox $1 \mu \mathrm{M}$ + 3-MA

$\square$ Dox $2.0 \mu \mathrm{M}$

$=\mathrm{Dox} 2.0 \mu \mathrm{M}+3-\mathrm{MA}$ b
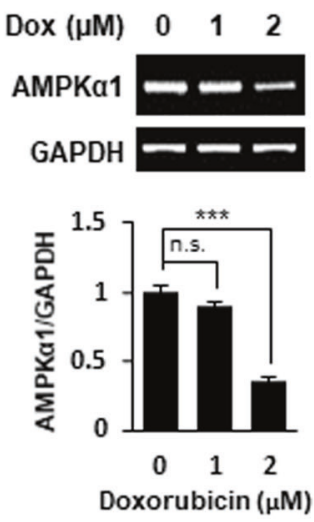

C
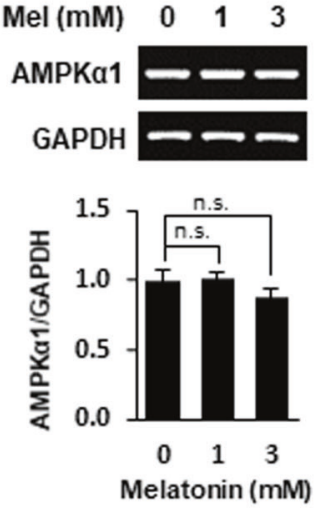

e
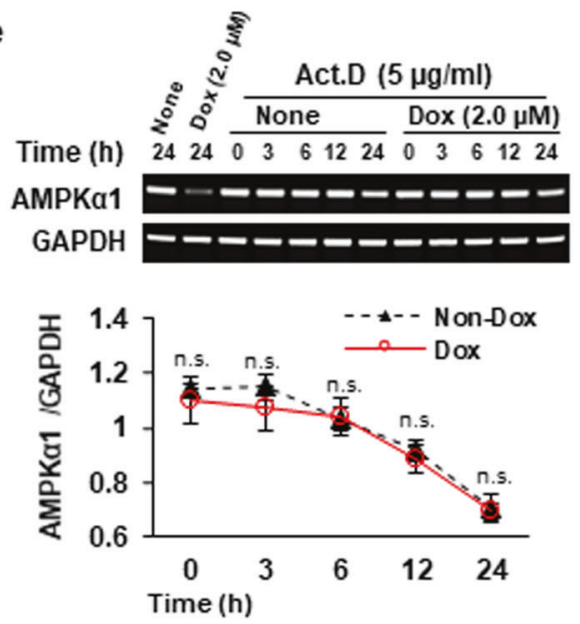

g

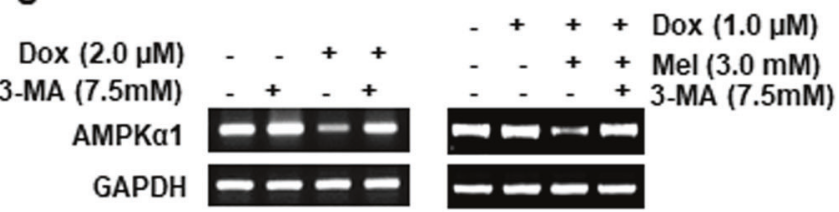

h

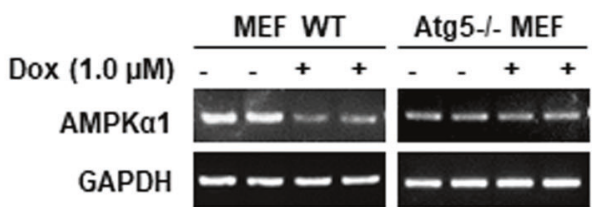

Fig. 4 Melatonin and doxorubicin synergistically reduce AMPKa1 at the transcriptional level. a MDA-MB-157 cells were cultured in the presence or absence of $2.0 \mu \mathrm{M}$ doxorubicin for the indicated time periods in the presence of cycloheximide. The protein levels of AMPK 1 were examined via Western blot analyses. b-d MDA-MB-157 cells were treated with the indicated concentrations of doxorubicin (b), melatonin (c), or a combination of $3 \mathrm{mM}$ melatonin and $1 \mu \mathrm{M}$ doxorubicin (d) for $24 \mathrm{~h}$. The cells were subjected to quantitative analysis of AMPK $\alpha 1$ mRNA. GAPDH served as a control. e MDA-MB-157 cells were cultured with or without doxorubicin $(2.0 \mu \mathrm{M})$ in the presence of actinomycin D (Act. D) for the indicated durations. The mRNA levels of AMPK $\alpha 1$ were analyzed. $\mathbf{f}$ MDA-MB-157 cells were transfected with a pGL3 luciferase reporter vector containing a human AMPK $\alpha 1$ promoter $(\sim 1.7 \mathrm{~kb})$. The cells were treated as indicated for $24 \mathrm{~h}$, and luciferase activity was measured. MDA-MB-157 cells (g) or wild-type and Atg $5^{-1-}$ MEFs (h) were treated as indicated for $24 \mathrm{~h}$, and the levels of AMPK $\alpha 1$ mRNA were measured. ${ }^{* * *} p<0.005 ;{ }^{* *} p<0.01 ;{ }^{*} p<0.05$; n.s. not significant. 
a

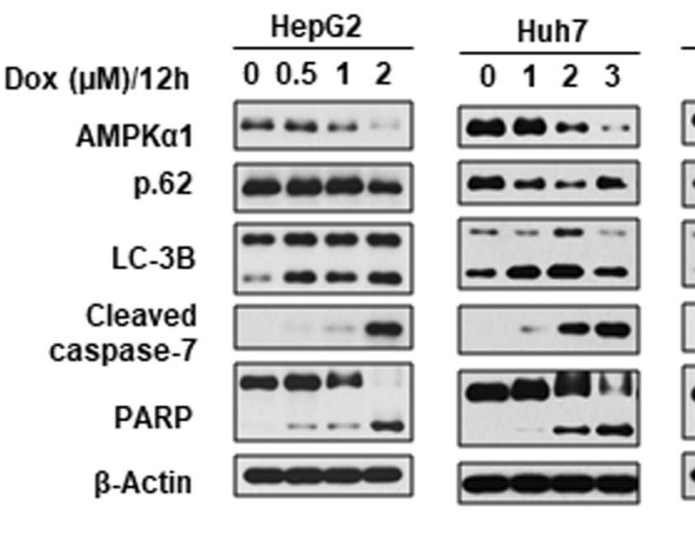

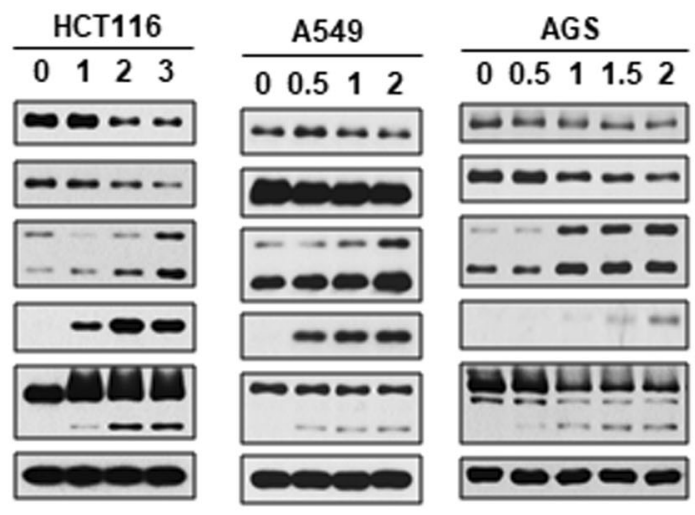

b
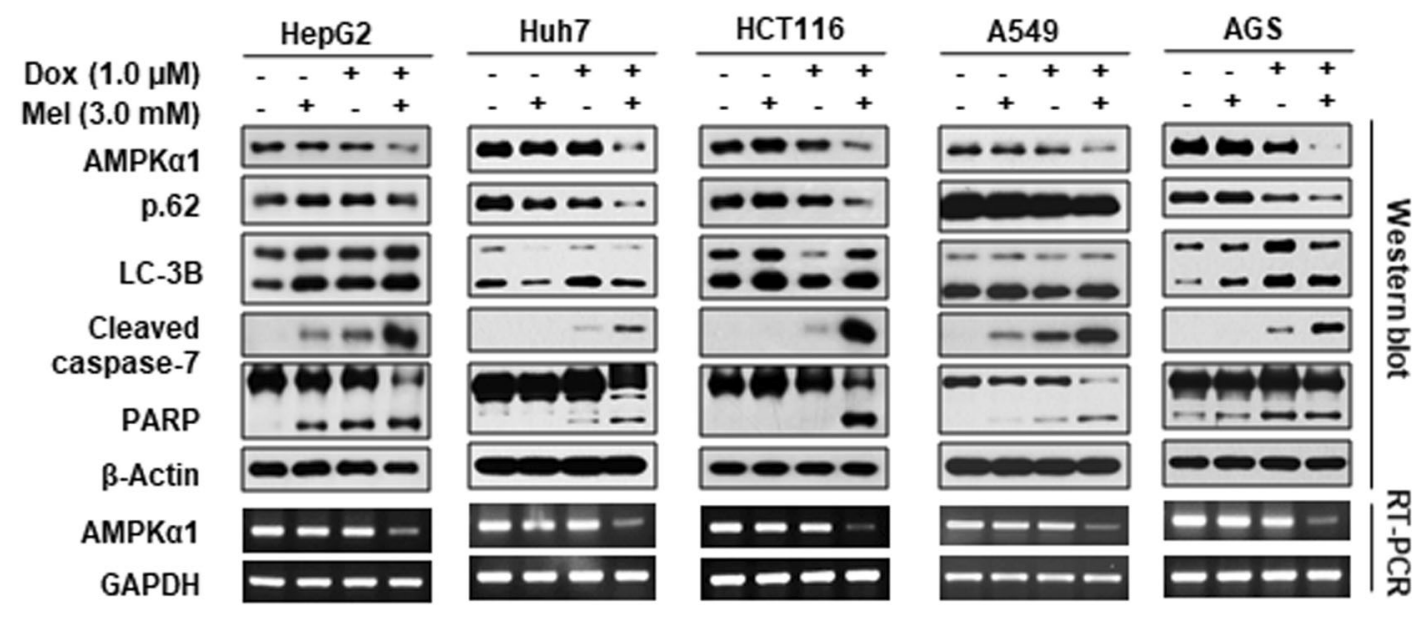

Fig. 5 The synergistic effects of melatonin and doxorubicin on apoptosis, autophagy, and AMPKa1 mRNA expression in different cancer cells. Hepatocellular carcinoma (HepG2, Huh7), colorectal carcinoma (HCT116), lung adenocarcinoma (A549), and gastric adenocarcinoma (AGS) cell lines were treated with the indicated concentration of doxorubicin (a) or with a combination of melatonin and doxorubicin (b) for $24 \mathrm{~h}$. The levels of the indicated proteins were examined via Western blot analysis (a, b), and the mRNA levels of AMPK 1 were examined by RT-PCR (b).

effects. However, the reduction in the expression of a survival factor such as AMPKa1 in cardiomyocytes may provide unfavorable environments for cancer patients, and a combination therapy involving melatonin and doxorubicin requires careful analyses of their effects on cardiomyocytes as well as on cancer cells.

Although the role of AMPK in metabolic regulation is quite well established, its roles under genotoxic stress or during tumorigenesis appear to be quite contradictory. First, there is no consensus regarding whether AMPK is activated ${ }^{26,27}$ or inhibited by doxorubicin ${ }^{28}$. Second, there are conflicting reports regarding the role of AMPK in tumor metabolism. Some studies have demonstrated that AMPK promotes tumor cell survival by regulating NADPH homeostasis ${ }^{35}$ or by balancing glycolysis and mitochondrial metabolism ${ }^{36}$. However, AMPK was also reported to suppress tumor growth by negatively regulating the Warburg effect $^{37}$. In the present study, we demonstrate that AMPKa1 acts as a survival factor for cancer cells in response to doxorubicin. In sharp contrast, AMPKa2 was reported to induce cell death under doxorubicin treatment in noncarcinoma cells ${ }^{29}$. AMPKa isoforms are therefore likely to exert opposing roles under genotoxic stress conditions. Given this, researchers should be careful to distinguish the roles of AMPKa isoforms when seeking to develop a novel chemotherapeutic strategy involving AMPK signaling pathways.

AMPK plays a central role as a cellular energy sensor in response to various conditions that alter the cellular energy level; the underlying mechanism involves rapid changes in its protein level via allosteric regulation ${ }^{23}$. Under ATP-depleting conditions, the accumulated AMP binds to the $\gamma$-subunit and activates AMPK. This interaction with AMP makes the kinase a better substrate for upstream kinases, leading to phosphorylation and the consequent full activation of AMPK. Intriguingly, we herein revealed that the level of AMPKa1 can be regulated at the transcriptional level in response to doxorubicin (Fig. 4). We previously reported that the transcription of AMPKa2 is robustly induced by E2F1 in noncarcinoma cells treated with doxorubicin ${ }^{29}$. Therefore, our present and previous results emphasize the complexity of AMPK regulatory mechanisms by revealing transcriptional regulation of AMPKa subunits. Additional work is needed to examine the mechanisms underlying the transcriptional regulation of AMPKa1.

Autophagy is generally considered to be a defense mechanism that maintains cell viability by recycling damaged cellular constituents under various conditions of cellular stress ${ }^{38}$. However, accumulating evidence suggests that excessive or dysregulated autophagy contributes to cell death ${ }^{39}$. It has also been reported that treatment with cytotoxic drugs, including doxorubicin, often results in the induction of autophagic cell death ${ }^{31,40}$, highlighting the complex signaling outcomes of autophagy. Accumulating data suggest that apoptosis-resistant cancer cells can undergo autophagy, and this induction of autophagic cell death has been suggested as a new strategy for cancer therapy ${ }^{30}$. Here, we observed that doxorubicin strongly induced autophagy that contributed to apoptosis; this apoptosis was significantly blocked in cells with defective autophagy (Fig. 3). Importantly, the mRNA levels and promoter activity of AMPKa1 were inhibited in an 
a

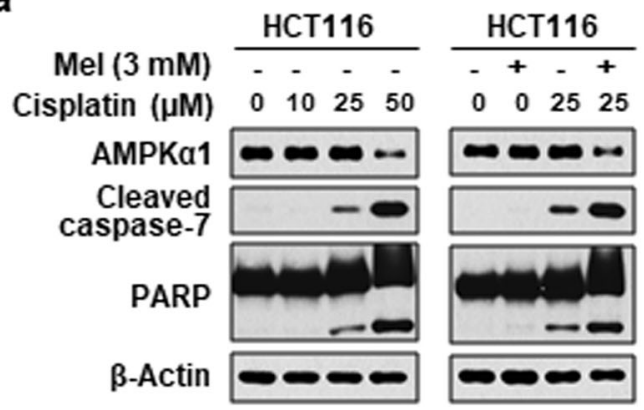

c

e

\begin{tabular}{rllll} 
& \multicolumn{3}{l}{ HCT116 } \\
\cline { 2 - 4 } Mel (3 mM) & - & + & - & + \\
Cisplatin $(\mu M)$ & 0 & 0 & 25 & 25 \\
AMPKa1 & - & - & - & - \\
GAPDH & & - & - & -
\end{tabular}

g

\begin{tabular}{|c|c|}
\hline \multirow{2}{*}{$\operatorname{Mel}(3 \mathrm{mM})$} & HCT116 \\
\hline & -+-+ \\
\hline notecan $(\mu \mathrm{M})$ & $\begin{array}{llll}0 & 0 & 10 & 1\end{array}$ \\
\hline AMPKa & --2 \\
\hline
\end{tabular}

b

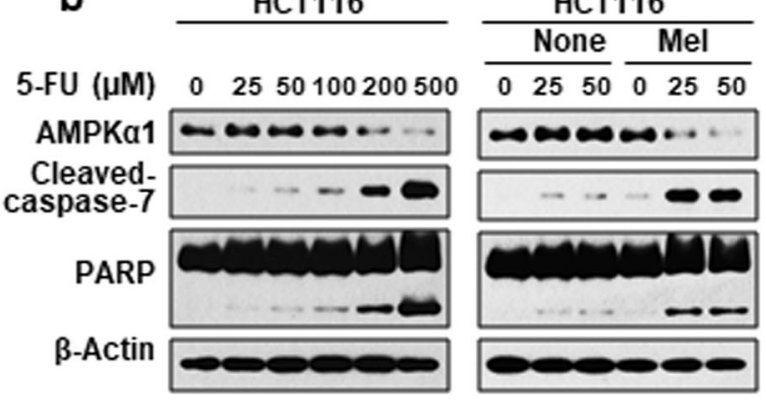

d

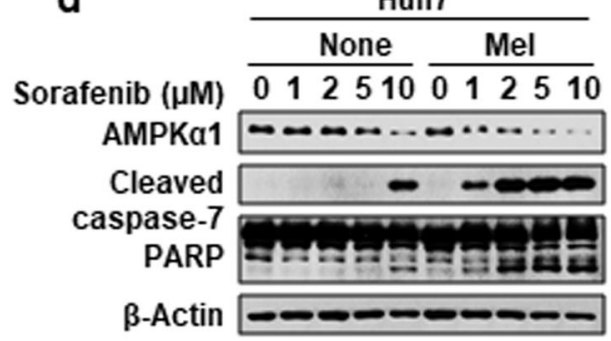

f

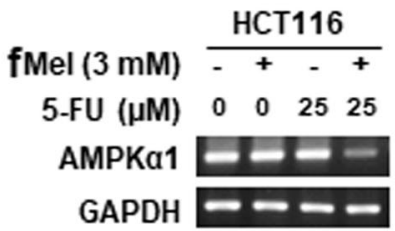

h

$\operatorname{Mel}(3 \mathrm{mM}) \frac{\text { Huh7 }}{-+-\quad+}$

Sorafenib ( $\mu \mathrm{M}) \quad \begin{array}{llll}0 & 0 & 2 & 2\end{array}$

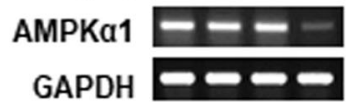

Fig. 6 The synergistic effects of melatonin with other anticancer drugs. HCT116 cells were treated with the indicated concentrations of cisplatin (a, e), 5-FU (b, f), and irinotecan $(\mathbf{c}, \mathbf{g})$ in the absence or presence of melatonin for $24 \mathrm{~h}$. Huh7 cells were treated with the indicated concentration of sorafenib in the absence or presence of melatonin for $24 \mathbf{h}(\mathbf{d}, \mathbf{h})$. Under these conditions, the levels of the indicated proteins (a-d) and mRNAs (e-h) were measured.

autophagy-dependent manner in our system (Fig. 4). To date, there is a lack of information on the specific transcription factor responsible for regulating the transcription of AMPKa1. Since the transcription of AMPKa1 was suppressed by doxorubicin in an autophagy-dependent manner, it would be highly intriguing to identify transcription factors that regulate AMPKa1 in an autophagydependent manner. For example, GATA4, which is a transcription factor known to be involved in cardiac development and function, is downregulated by doxorubicin ${ }^{41}$ and subjected to autophagydependent degradation ${ }^{42}$. We indeed examined this possibility, but GATA4 is unlikely to regulate the AMPKa1 promoter (Supplementary Fig. 1). Nevertheless, we cannot completely rule out the involvement of GATA4 because a relatively short region of the AMPKa1 promoter $(\sim 1.7 \mathrm{~kb})$ was examined in this study. Further studies on the regulation of the AMPKa1 promoter would provide us with a highly intriguing insight into not only the regulatory mechanisms of AMPK but also potential applications for chemotherapy.
In conclusion, we herein suggest that AMPKa1 is a common survival factor for various cancer cells, that a reduction in AMPKa1 expression can be exploited as an adjuvant therapy to render many cancer cell types sensitive to anticancer drugs, and that this reduction further alleviates the adverse effects of anticancer drugs.

\section{REFERENCES}

1. Torre, L. A. et al. Global cancer statistics, 2012. CA A Cancer J. Clin. 65, 87-108 (2015).

2. Harbeck, N. \& Gnant, M. Breast cancer. Lancet 389, 1134-1150 (2017).

3. Movahedi, M. et al. Survival rate of breast cancer based on geographical variation in iran, a national study. Iran. Red. Crescent Med. J. 14, 798-804 (2012).

4. Crozier, J. A., Swaika, A. \& Moreno-Aspitia, A. Adjuvant chemotherapy in breast cancer: to use or not to use, the anthracyclines. World J. Clin. Oncol. 5, 529-538 (2014).

5. Seddon, B. et al. Gemcitabine and docetaxel versus doxorubicin as first-line treatment in previously untreated advanced unresectable or metastatic soft- 
tissue sarcomas (GeDDiS): a randomised controlled phase 3 trial. Lancet Oncol. 18, 1397-1410 (2017)

6. Judson, I. et al. Doxorubicin alone versus intensified doxorubicin plus ifosfamide for first-line treatment of advanced or metastatic soft-tissue sarcoma: a randomised controlled phase 3 trial. Lancet Oncol. 15, 415-423 (2014).

7. Hosseini, A. \& Sahebkar, A. Reversal of doxorubicin-induced cardiotoxicity by using phytotherapy: a review. J. Pharmacopunct. 20, 243-256 (2017).

8. Reiter, R. J. Pineal melatonin: cell biology of its synthesis and of its physiological interactions. Endocr. Rev. 12, 151-180 (1991).

9. Su, S. C. et al. Cancer metastasis: mechanisms of inhibition by melatonin. J. Pineal Res. 62, e12370 (2017).

10. $\mathrm{Li}, \mathrm{W}$. et al. Melatonin treatment induces apoptosis through regulating the nuclear factor-kappaB and mitogen-activated protein kinase signaling pathways in human gastric cancer SGC7901 cells. Oncol. Lett. 13, 2737-2744 (2017).

11. Li, W. et al. Melatonin induces cell apoptosis in Mia PaCa-2 cells via the suppression of nuclear factor-kappaB and activation of ERK and JNK: a novel therapeutic implication for pancreatic cancer. Oncol. Rep. 36, 2861-2867 (2016).

12. Wei, J. Y., Li, W. M., Zhou, L. L., Lu, Q. N. \& He, W. Melatonin induces apoptosis of colorectal cancer cells through HDAC4 nuclear import mediated by CaMKII inactivation. J. Pineal Res. 58, 429-438 (2015).

13. Li, W. et al. Melatonin induces cell apoptosis in AGS cells through the activation of JNK and P38 MAPK and the suppression of nuclear factor-kappa B: a novel therapeutic implication for gastric cancer. Cell. Physiol. Biochem. 37, 2323-2338 (2015).

14. Li, Z. et al. Melatonin inhibits nucleus pulposus (NP) cell proliferation and extracellular matrix $(E C M)$ remodeling via the melatonin membrane receptors mediated PI3K-Akt pathway. J. Pineal Res. 63, e12345 (2017).

15. Sanchez-Hidalgo, M., Lee, M., de la Lastra, C. A., Guerrero, J. M. \& Packham, G. Melatonin inhibits cell proliferation and induces caspase activation and apoptosis in human malignant lymphoid cell lines. J. Pineal Res. 53, 366-373 (2012).

16. Lin, S. et al. Melatonin promotes sorafenib-induced apoptosis through synergistic activation of JNK/c-jun pathway in human hepatocellular carcinoma. J. Pineal Res. 62, e12398 (2017)

17. Fan, L. L. et al. Melatonin and doxorubicin synergistically induce cell apoptosis in human hepatoma cell lines. World J. Gastroenterol. 16, 1473-1481 (2010).

18. Gao, Y. et al. Melatonin synergizes the chemotherapeutic effect of 5-fluorouracil in colon cancer by suppressing PI3K/AKT and NF-kappaB/iNOS signaling pathways. J. Pineal Res. 62, e12380 (2016).

19. Pariente, R., Pariente, J. A., Rodriguez, A. B. \& Espino, J. Melatonin sensitizes human cervical cancer HeLa cells to cisplatin-induced cytotoxicity and apoptosis: effects on oxidative stress and DNA fragmentation. J. Pineal Res. 60, 55-64 (2016).

20. Kim, J. H. et al. Melatonin synergistically enhances cisplatin-induced apoptosis via the dephosphorylation of ERK/p90 ribosomal S6 kinase/heat shock protein 27 in SK-OV-3 cells. J. Pineal Res. 52, 244-252 (2012).

21. Prieto-Dominguez, N. et al. Melatonin-induced increase in sensitivity of human hepatocellular carcinoma cells to sorafenib is associated with reactive oxygen species production and mitophagy. J. Pineal Res. 61, 396-407 (2016).

22. Reiter, R. J., Tan, D. X., Sainz, R. M., Mayo, J. C. \& Lopez-Burillo, S. Melatonin: reducing the toxicity and increasing the efficacy of drugs. J. Pharm. Pharmacol. 54, 1299-1321 (2002)

23. Hardie, D. G. Sensing of energy and nutrients by AMP-activated protein kinase. Am. J. Clin. Nutr. 93, 891S-896S (2011).

24. Hardie, D. G. AMPK: positive and negative regulation, and its role in whole-body energy homeostasis. Curr. Opin. Cell Biol. 33, 1-7 (2015).

25. Stapleton, D. et al. Mammalian AMP-activated protein kinase subfamily. J. Biol. Chem. 271, 611-614 (1996).

26. Chen, M. B. et al. Activation of AMP-activated protein kinase contributes to doxorubicin-induced cell death and apoptosis in cultured myocardial H9c2 cells. Cell Biochem. Biophys. 60, 311-322 (2011).

27. Ji, C. et al. Exogenous cell-permeable C6 ceramide sensitizes multiple cancer cell lines to doxorubicin-induced apoptosis by promoting AMPK activation and mTORC1 inhibition. Oncogene 29, 6557-6568 (2010).

28. Wang, S., Song, P. \& Zou, M. H. Inhibition of AMP-activated protein kinase alpha (AMPKalpha) by doxorubicin accentuates genotoxic stress and cell death in mouse embryonic fibroblasts and cardiomyocytes: role of p53 and SIRT1. J. Biol. Chem. 287, 8001-8012 (2012).

29. Yang, W. et al. AMP-activated protein kinase alpha2 and E2F1 transcription factor mediate doxorubicin-induced cytotoxicity by forming a positive signal loop in mouse embryonic fibroblasts and non-carcinoma cells. J. Biol. Chem. 289, 4839-4852 (2014)

30. Chen, C. et al. Autophagy and doxorubicin resistance in cancer. Anticancer Drugs 29, 1-9 (2018)

31. Wei, T., Xiaojun, X. \& Peilong, C. Magnoflorine improves sensitivity to doxorubicin (DOX) of breast cancer cells via inducing apoptosis and autophagy through AKT/ mTOR and p38 signaling pathways. Biomed. Pharmacother. 121, 109139 (2020).
32. Najafi, M., Hooshangi Shayesteh, M. R., Mortezaee, K., Farhood, B. \& HaghiAminjan, $\mathrm{H}$. The role of melatonin on doxorubicin-induced cardiotoxicity: a systematic review. Life Sci. 241, 117173 (2020).

33. Kosar, P. A., Naziroglu, M., Ovey, I. S. \& Cig, B. Synergic effects of doxorubicin and melatonin on apoptosis and mitochondrial oxidative stress in MCF-7 breast cancer cells: involvement of TRPV1 channels. J. Membr. Biol. 249, 129-140 (2016).

34. Liu, X. et al. Melatonin as an effective protector against doxorubicin-induced cardiotoxicity. Am. J. Physiol. Heart Circ. Physiol. 283, H254-H263 (2002).

35. Jeon, S. M., Chandel, N. S. \& Hay, N. AMPK regulates NADPH homeostasis to promote tumour cell survival during energy stress. Nature 485, 661-665 (2012).

36. Kishton, R. J. et al. AMPK is essential to balance glycolysis and mitochondrial metabolism to control T-ALL cell stress and survival. Cell Metab. 23, 649-662 (2016).

37. Faubert, B. et al. AMPK is a negative regulator of the Warburg effect and suppresses tumor growth in vivo. Cell Metab. 17, 113-124 (2013).

38. D'Arcy, M. S. Cell death: a review of the major forms of apoptosis, necrosis and autophagy. Cell Biol. Int. 43, 582-592 (2019).

39. Mathew, R., Karantza-Wadsworth, V. \& White, E. Role of autophagy in cancer. Nat. Rev. Cancer 7, 961-967 (2007).

40. Lin, L. T. et al. Paris polyphylla inhibits colorectal cancer cells via inducing autophagy and enhancing the efficacy of chemotherapeutic drug doxorubicin. Molecules 24, 2102 (2019).

41. Park, A. M. et al. Mechanism of anthracycline-mediated down-regulation of GATA4 in the heart. Cardiovasc. Res. 90, 97-104 (2011).

42. Kang, $C$. et al. The DNA damage response induces inflammation and senescence by inhibiting autophagy of GATA4. Science 349, aaa5612 (2015).

\section{AUTHOR CONTRIBUTIONS}

Conceptualization, W.C., I.K., S.S.K., J.H.; investigation, Q.H.T., D.H.H., M.S.; writingoriginal draft preparation, Q.H.T., J.H. All authors have read and agreed to the published version of the manuscript.

\section{FUNDING INFORMATION}

This work was supported by grants from the National Research Foundation of Korea (NRF-2020R1F1A1049980) and the Basic Science Research Program through the National Research Foundation of Korea (NRF) funded by the Ministry of Education (2018R1A6A1A03025124).

\section{COMPETING INTERESTS}

The authors declare no competing interests.

\section{ADDITIONAL INFORMATION}

Supplementary information The online version contains supplementary material available at https://doi.org/10.1038/s12276-021-00675-y.

Correspondence and requests for materials should be addressed to Joohun $\mathrm{Ha}$.

Reprints and permission information is available at http://www.nature.com/ reprints

Publisher's note Springer Nature remains neutral with regard to jurisdictional claims in published maps and institutional affiliations.

Open Access This article is licensed under a Creative Commons Attribution 4.0 International License, which permits use, sharing, adaptation, distribution and reproduction in any medium or format, as long as you give appropriate credit to the original author(s) and the source, provide a link to the Creative Commons license, and indicate if changes were made. The images or other third party material in this article are included in the article's Creative Commons license, unless indicated otherwise in a credit line to the material. If material is not included in the article's Creative Commons license and your intended use is not permitted by statutory regulation or exceeds the permitted use, you will need to obtain permission directly from the copyright holder. To view a copy of this license, visit http://creativecommons. org/licenses/by/4.0/.

(c) The Author(s) 2021 